\title{
SITUACIÓN DE TRES RAZAS AUTÓCTONAS ESPAÑOLAS DE LA ESPECIE OVINA EN CASTILLA Y LEÓN
}

\author{
SITUATION OF THREE AUTOCHTHONOUS SPANISH RACES OF THE SHEEP \\ SPECIES IN CASTILLA Y LEÓN \\ Yanes, J.E. ${ }^{1}$ y de la Fuente, L.F. ${ }^{2}$ \\ 'Servicio Territorial de Agricultura y Ganadería de Zamora. Zamora. España. yangarjo@jcyl.es \\ ${ }^{2}$ Departamento de Producción Animal. Facultad de Veterinaria. Universidad de León. León. España. \\ Palabras clave adicionales \\ Censo. Castellana. Churra. Ojalada. \\ AdDitiONAL KEYWORDS \\ Census. Castellana. Churra. Ojalada.
}

\section{RESUMEN}

En tres razas autóctonas españolas (Churra, Castellana y Ojalada) se estudió la situación censal, incluyendo su orientación productiva, tanto de explotaciones como de animales, así como su participación en dos denominaciones de calidad y la inscripción en sus libros genealógicos.

Los mayores censos de animales correspondieron a la raza Churra seguidos de la Castellana, en ambas con predominio de los rebaños de orientación carne; a mucha mayor distancia se encontraron los de la Ojalada.

La inscripción de explotaciones en la DO Queso Zamorano, a tenor de su orientación productiva, fue prácticamente total para el caso de la raza Castellana. Para la IGP Lechazo de Castilla y León el número de explotaciones inscritas en la misma fue bajo, excepto en la raza Ojalada.

Las inscripciones en los libros genealógicos resultaron muy bajas, a excepción de la raza Ojalada.

\section{SUMMARY}

In three autochthonous spanish races (Churra, Castellana and Ojalada) studied the sensual situation, including his productive orientation, both of developments and of animals, as well as his participation in two denominations of quality and the inscription in his genealogical books.

The major censuses of animals corresponded to the race Churra followed by the Castellana, in

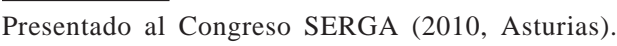

both with predominance of the flocks of orientation meat; to many major distance those of the Ojalada were.

The inscription of developments in the DO Queso Zamorano, to tenor of his productive orientation, was practically total for the case of the Castellana race. For the IGP Lechazo de Castilla y León the number of developments inscribed in the same one was low, except in the race Ojalada.

The inscriptions in the genealogical books turned out to be very low, with the exception of the race Ojalada.

\section{INTRODUCCIÓN}

La Comunidad Autónoma de Castilla y León posee un rico patrimonio zoogenético representado por una amplia variedad de razas autóctonas, entre ellas tres ovinas, destacando como emblemáticas la Churra y la Castellana (ambas de doble aptitud u orientación productiva). La Ojalada y la variedad negra de la Castellana se hallan catalogadas como razas autóctonas en peligro de extinción.

Los últimos censos oficiales al respecto datan del año 1986, siendo publicados en el Anuario de Estadística Agraria del entonces Ministerio de Agricultura, Pesca y Alimentación, atribuyéndosele 1770230 animales para la raza Churra, 1650000 para la 


\section{YANES Y DE LA FUENTE}

raza Castellana y 470784 para la raza Ojalada. Datos desde hace muchos años totalmente obsoletos, no solo en lo que se refiere a la cuestión numérica sino también al área geográfica de desenvolvimiento de las tres razas, hoy limitado al ámbito de Castilla y León (de la Fuente, 2006). Tampoco existen en la actualidad bases de datos específicas para obtener los censo de razas autóctonas, salvo los inscritos en los libros genealógicos.

La DO Queso Zamorano (en adelante DO) ampara la fabricación de quesos fabricados con leche exclusivamente de las razas Churra y Castellana de la provincia de Zamora, sin embargo la IGP Lechazo de Castilla y León (en adelante IGP) ampara lechazos procedentes de las tres razas localizadas en una gran parte del territorio de Castilla y León.

El objetivo del estudio, a 31/12/08, es evaluar el censo de las tres razas ovinas desde el ámbito provincial, así como su orientación productiva (Churra y Castellana). Se ha estudiado el alcance de las inscripciones en las dos denominaciones de calidad y realizado un análisis de las inscripciones en los libros genealógicos respecto a la situación censal de cada una de ellas.

\section{MATERIAL YMÉTODOS}

Para el estudio censal de las razas Churra y Castellana y su orientación productiva, se elaboró un documento al efecto que fue distribuida entre las 103 Unidades Veterinarias de Castilla y León para su cumplimentación en base a los conocimientos in situ. Se compararon los datos obtenidos con las bases de datos de Saneamiento Ganadero y del Registro de Explotaciones Ganaderas donde figuran la raza y orientación productiva, realizando un exhaustiva contrastación de los mismos. Obtenidos los censos provinciales de las tres razas, se compararon con los censos totales de la especie ovina.

De los dos Consejos Reguladores de las denominaciones de calidad se obtuvieron datos de las tres razas con el fin de proceder a su estudio y determinar su participación en las mismas.

Las inscripciones en los libros genealógicos fueron obtenidas de las asociaciones de criadores, a través del diseño de un modelo específico donde se incluyeron las orientaciones productivas.

Tanto en las denominaciones de calidad como en los libros genealógicos se compararon los datos obtenidos con los censos de cada raza.

\section{RESULTADOSYDISCUSIÓN}

Los censos provinciales para las tres razas pueden observarse en la tabla I (Yanes, 2008). La raza Churra se halla presente en la provincia de Burgos con el $56 \%$ de los animales y el 67,6\% de las explotaciones, siguiéndole a distancia las provincias de León (12,2\% y 12,3\%), Palencia $(10,1 \%$ y $5,3 \%)$, Zamora ( $8,9 \%$ y $7 \%)$, Segovia $(5,1 \%$ y $3,8 \%$ ) y Valladolid $(4,2 \%$ y $2,3 \%)$, y censos testimoniales en Soria, Salamanca y Ávila. El $62,3 \%$ de los animales y el 74,4\% de las explotaciones son de orientación carnicera.

La raza Castellana se halla fundamentalmente en la provincia de Zamora, con el $68,6 \%$ de los animales y el $66,7 \%$ de las explotaciones (comarcas del centro y oeste), dedicándose más del $91 \%$ de su censo a la orientación carnicera. De la variedad negra hay 40000 animales repartidos en sus comarcas de Tábara (38,3\%), Aliste (28,3\%), Alba $(25,2)$, Sayago $(20,5)$ y Carballeda $(18,5 \%)$, con una media de un $26,7 \%$ de los animales/ rebaño, aunque con rebaños que superan el $30 \%$.

En la raza Ojalada el 82,4\% de las explotaciones y el $92,3 \%$ de los animales se localiza en la provincia de Soria, mayoritariamente en el sur y sureste de la misma, aunque dos ganaderías poseen el $41,7 \%$ de los animales.

El censo de las tres razas autóctonas representa el 19,82\% de las explotaciones y el 19,20\% de los animales de la especie ovina de Castilla y León (Churra 13,04\%, Castella- 
Tabla I. Censo de la razas autóctonas ovinas de Castilla y León. (Census of the autochthonous ovine breeds of Castilla y León).

\begin{tabular}{|c|c|c|c|c|c|c|c|c|}
\hline \multirow[t]{2}{*}{ Provincia } & \multicolumn{2}{|c|}{ Churra } & \multicolumn{2}{|c|}{ Castellana } & \multicolumn{2}{|c|}{ Ojalada } & \multicolumn{2}{|c|}{ Ovino autóctono } \\
\hline & Expl. & Anim. & Expl. & Anim. & Expl. & Anim. & Expl. & Anim. \\
\hline Zamora & 119 & 42618 & 422 & 149634 & 0 & 0 & 541 & 192252 \\
\hline Salamanca & 8 & 5754 & 50 & 19000 & 1 & 86 & 59 & 24840 \\
\hline León & 209 & 58092 & 2 & 2500 & 0 & 0 & 211 & 60592 \\
\hline Valladolid & 39 & 19940 & 56 & 18779 & 0 & 0 & 95 & 38719 \\
\hline Segovia & 65 & 24452 & 30 & 12018 & 2 & 594 & 97 & 37064 \\
\hline Soria & 13 & 7150 & 30 & 11256 & 14 & 8224 & 57 & 26630 \\
\hline Burgos & 1144 & 266550 & 1 & 250 & 0 & 0 & 1145 & 266800 \\
\hline Palencia & 90 & 47982 & 2 & 1748 & 0 & 0 & 92 & 49730 \\
\hline Ávila & 6 & 3715 & 22 & 922 & 0 & 0 & 28 & 4637 \\
\hline TOTAL & 1693 & 476253 & 615 & 224407 & 17 & 8904 & 2325 & 701264 \\
\hline
\end{tabular}

Censos a $31 / 12 / 08$.

na $6,14 \%$ y Ojalada $0,24 \%$ ).

En la DO se hallan inscritas 92 explotaciones y 45150 animales: el 100\% de la raza Castellana y el $60 \%$ de las explotaciones y $58,5 \%$ de los animales de Churra. De los 123799 lechazos sacrificados como aptos bajo la IGP, el 70,8\% corresponde a la Churra, el $24,9 \%$ a la Castellana y el $4,3 \%$ a la Ojalada.

En la raza Churra las inscripciones en el libro genealógico fueron 317 explotaciones y 171923 animales, es decir, un $18,8 \%$ de las explotaciones y un $36,1 \%$ de los animales de la raza, con mayoría de explotaciones de orientación carne $(60,3 \%)$, aunque venía siendo tradicional que lo fueran de ordeño. Las inscripciones para la Castellana ascendieron a 53 ganaderías con un total de 33077 animales, es decir, tan solo el $8,6 \%$ de las explotaciones y el $14,7 \%$ de los animales de la raza; casi el $80 \%$ de las explotaciones son de orientación carne.

Los censos de las razas Churra y Castellana se hallan realmente muy por debajo de lo que habitualmente se le atribuye (Anuario Estadístico de Castilla y León 2007). Ambas razas, de doble aptitud, se dedican mayoritariamente a producir solo lechazos en un porcentaje muy similar de explotaciones, sin embargo la raza Castellana lo hace sobre mayor porcentaje de animales.

El número potencial de explotaciones que pueden inscribirse en la DO es bajo (120), sin embargo es muy alto en el caso de la IGP (2325), en ambos casos influye su ámbito geográfico aunque de forma diferente.

La inscripción de explotaciones en los libros genealógicos debería ser tomada en mayor consideración por las Administraciones para la recepción de cualquier tipo de ayudas a razas autóctonas por parte de los ganaderos.

\section{CONCLUSIONES}

En Castilla y León las razas autóctonas ovinas Churra, Castellana y Ojalada son minoritarias en comparación con el censo total de la especie, sin embargo, las dos primeras tienen aún una gran significación en la economía y tradición ganadera, con independencia de su gran valor medioambiental.

El mayor censo corresponde a la raza Churra, localizada sobre todo en la provincia de Burgos, y orientada mayoritariamente 


\section{YANES Y DE LA FUENTE}

hacia la producción exclusiva de lechazos. Sin embargo, en las provincias de Valladolid y de Zamora su menor censo de dedica a producción lechera.

La raza Castellana se explota y concentra en la provincia de Zamora (comarcas naturales del centro y oeste), al igual que la variedad negra, dedicándose en muy alto porcentaje a la producción de lechazos, siendo en ésta provincia también donde se encuentran las minoritarias explotaciones lecheras.

El desplome actual de la raza Ojalada es impresionante, debido a lo reducido del número de rebaños, animales y agravado por la concentración de éstos en tan solo dos explotaciones. Se localiza en la provincia de Soria a excepción de tres rebaños.

En la DO se halla inscrito un censo muy elevado de animales de raza Castellana y Churra de orientación lechera en la provincia de Zamora (la totalidad en el caso de la raza Castellana), debido a su obligatoriedad para comercializar leche con destino a la DO Queso Zamorano. Se sacrifica un porcenta-

\section{BIBLIOGRAFÍA}

Anuario Estadístico de Castilla y León. 2007. Junta de Castilla y León. D.G. de Estadística. León. $753 \mathrm{pp}$.

De la Fuente, L.F. 2006. La oveja del futuro. je muy bajo de lechazos (5\%) al amparo de la IGP ya que no reperesnta para el ganadero una gran ventaja económica respecto a otros cauces de comercialización, siendo la Ojalada la que tiene el mayor porcentaje de explotaciones inscritas en relación a su censo racial.

Hay un porcentaje bajo de explotaciones y animales inscritos en los libros genealógicos de las razas Churra y Castellana (mucho menor en ésta última), sin embargo, en la Ojalada se encuentran inscritos la totalidad.

\section{AGRADECIMIENTOS}

A la Dirección General de Producción Agropecuaria, y a los Servicios Veterinarios Oficiales de la Consejería de Agricultura y Ganadería por el esfuerzo realizado en la obtención de datos.

A los Servicios Técnicos de las asociaciones de criadores de las tres razas autóctonas ovinas de Castilla y León (ANCHE, ANCA y ANCRO) por la eficiencia demostrada.

Tierras de Castilla y León, 126: 8-13.

Yanes, J.E. 2008. La Castellana y la Churra, dos razas emblemáticas de Castilla y León. Situación actual. FEAGAS, 33: 70-77. 